\title{
Anatomical observation and clinical significance of the left gastric vein in laparoscopic radical gastrectomy
}

\author{
Xiaofeng Zhu ${ }^{1,2 \#}$, Qiang Zhao ${ }^{3 \#}$, Wenjun Xiong ${ }^{4,5}$, Lijie Luo ${ }^{4,5}$, Yansheng Zheng ${ }^{4,5}$, Haipeng Huang ${ }^{4,5}$, \\ Jin $\mathrm{Li}^{4,5}$, Jin Wan ${ }^{4,5}$, Wei Xie ${ }^{6}$, Wei Wang ${ }^{4,5}$ \\ ${ }^{1}$ Department of Vascular Intervention, Guangdong Provincial Hospital of Chinese Medicine, Guangzhou, China; ${ }^{2}$ Department of Vascular \\ Intervention, The 2nd Clinical College of Guangzhou University of Chinese Medicine, Guangzhou, China; ${ }^{3}$ Department of Surgery, Taishan \\ Hospital of Traditional Chinese Medicine, Jiangmen, China; ${ }^{4}$ Department of Gastrointestinal Surgery, Guangdong Provincial Hospital of Chinese \\ Medicine, Guangzhou, China; ${ }^{5}$ Department of Gastrointestinal Surgery, The 2nd Clinical College of Guangzhou University of Chinese Medicine, \\ Guangzhou, China; ${ }^{6}$ Department of Gastrointestinal Surgery, People's Hospital of Yangjiang, Yangjiang, China \\ Contributions: (I) Conception and design: W Wang, W Xie; (II) Administrative support: W Wang; (III) Provision of study materials or patients: W \\ Wang, W Xiong; (IV) Collection and assembly of data: X Zhu, Q Zhao; (V) Data analysis and interpretation: W Xiong; (VI) Manuscript writing: All \\ authors; (VII) Final approval of manuscript: All authors. \\ \#These authors contributed equally to this work. \\ Correspondence to: Prof. Wei Wang, MD. Department of Gastrointestinal Surgery, Guangdong Provincial Hospital of Chinese Medicine, the Second \\ Affiliated Hospital of Guangzhou University of Chinese Medicine, Dade Road No.111, Guangzhou 510120, China. Email: wangwei16400@163.com; \\ Wei Xie, MD. Department of Gastrointestinal Surgery, People's Hospital of Yangjiang, Dongshan South Road No.42, Yangjiang 529500, China. \\ Email: m13902528731@126.com.
}

Background: The left gastric vein (LGV) plays an important role in laparoscopic radical gastrectomy (LRG). However, the anatomy of the LGV is complicated with significant variation, and it is often damaged and bleeding during LRG. The purpose of this study was to observe and analyze the anatomic types of the LGV in patients undergoing LRG and to explore its clinical significance.

Methods: A total of 217 patients who underwent LRG from June 2016 to December 2020 were included. LGVs were divided into four types according to the relationship between the LGV and peripheral arteries [celiac artery (CA)/common hepatic artery (CHA)/splenic artery (SA)] and the pancreas during LRG. If a LGV was damaged during surgery (resulting in bleeding), it was included in the bleeding group. Nonbleeding groups were included if there was no impairment to the LGV.

Results: A total of four types of LGVs were observed, of which type I was the most prevalent, accounting for $58.8 \%(\mathrm{n}=121)$. In 21 patients $(9.7 \%)$, the LGV was injured and hemorrhagic during LRG; and the type IV LGV injury bleeding rate was as high as $41.7 \%(5 / 12)$. Univariate analysis revealed that the extent of lymph node dissection (LND), pathological stage, tumor (T) stage, and type of LGV were significantly associated with LGV injury and hemorrhage $(\mathrm{P}<0.05)$. Multivariate analysis showed that enlarged LND, late T stage, late pathological stage, and type IV LGV were independent risk factors for LGV injury hemorrhage. Conclusions: LGVs that run between the CHA (posterior) and the CA into the portal venous system were the most common anatomical type. A LGV that runs between the SA (posterior) and the CA into the portal venous system is easily injured (resulting in bleeding). LGV injury and hemorrhage are affected by a variety of factors, and therefore, careful intraoperative dissection is necessary to avoid damage to the LGV.

Keywords: Left gastric vein (LGV); laparoscopic radical gastrectomy (LRG); anatomical observation; clinical significance

Submitted Jun 04, 2021. Accepted for publication Jul 29, 2021.

doi: 10.21037/jgo-21-352

View this article at: https://dx.doi.org/10.21037/jgo-21-352 


\section{Introduction}

In China, gastric cancer is a common malignant tumor of the digestive tract, and surgery is the main method used to treat advanced gastric cancer. In recent years, laparoscopic radical gastrectomy (LRG) has become a common method for the treatment of gastric cancer worldwide, with the advantages of light pain, small trauma and quick recovery (1-3). However, anatomic variations of perigastric vascular structures increase the difficulty of laparoscopic surgery, and the lack of tactile sensation in laparoscopic surgery increases the risk of vascular injury, particularly venous injury $(4,5)$.

The left gastric vein (LGV), also known as the coronary gastric vein, is one of the important vessels requiring dissection in LRG, and is usually incorporated into the portal vein $(\mathrm{PV})$ or splenic vein $(\mathrm{SV})(5,6)$. Therefore, accidental injury to the LGV during surgery can result in significant blood loss. Previous studies of the LGV have focused on imaging and anatomy, and lack physical observation. At present, there is relatively small guidance for laparoscopic surgery. Therefore, laparoscopic anatomical classification of the LGV has a direct guiding significance for surgery, which can quickly improve the level of laparoscopic surgery among young physicians and provide greater benefits to patients. In this study, we observed and analyzed the anatomical classification of the LGV in patients undergoing LRG and explored its clinical significance. We present the following article in accordance with the STROBE reporting checklist (available at https:// dx.doi.org/10.21037/jgo-21-352).

\section{Methods}

\section{Patients}

This retrospective study aimed to observe and analyze 230 LRG videos stored at the Department of Gastrointestinal Surgery, Guangdong Provincial Hospital of Chinese Medicine, from January 2016 to December 2020. Of these, six patients were found to have LGV deficiency intraoperatively, and another six were found to have extensive adhesions or intraoperative major hemorrhage and open laparotomy. In one patient, the common hepatic artery (CHA) was not found during the operation. A total of 217 patients were included in this study. All procedures performed in this study involving human participants were in accordance with the Declaration of Helsinki (as revised in 2013). The study was approved by regional ethics board of
Guangdong Provincial Hospital of Chinese Medicine (NO: ZE2021-173-01). Individual consent for this retrospective analysis was waived.

\section{Operating procedure}

LRG involved laparoscopic distal, proximal, and total gastrectomy, with D2 to D2+ lymph node dissection (LND) performed according to the third Japanese gastric cancer treatment guidelines (7). All surgical procedures were recorded on a laparoscopic video system, and were performed by a deputy chief physician (or above) in our department.

\section{Classification and nomenclature of the $L G V$ variants}

LGVs in this study were divided into four types according to the relationship between the $\mathrm{LGV}$ and peripheral arteries [celiac artery (CA), CHA, and splenic artery (SA)] and the pancreas during LRG. Type I: the LGV runs between CHA (posterior) and CA into the portal venous system. Type II: the LGV runs between the pancreatic body (posterior) and the CHA (anterior) into the portal venous system. Type III: the LGV runs between the pancreatic body (posterior) and the SA (anterior) into the portal venous system. Type IV: the LGV runs between the SA (posterior) and the CA into the portal venous system. If a LGV was damaged during surgery (resulting in bleeding), it was included in the bleeding group. Non-bleeding groups were included if there was no intraoperative bleeding. If the patient had two or more LGVs, the type of LGV was determined by the largest branch.

\section{Statistical analysis}

Data management and statistical analyses were performed using IBM SPSS Statistics (Version 20.0; IBM Corp., New York, USA) and R software (Version 4.0.3; R Foundation for Statistical Computing, Vienna, Austria). Counting data were expressed as rates, and the $\chi^{2}$ test was performed. Measurement data were expressed as $\bar{x} \pm \mathrm{s}$, and the test was performed. The logistic regression model was used to analyze the risk factors of LGV injury. $\mathrm{P}<0.05$ was considered statistically significant.

\section{Results}

The general data, pathological characteristics, and surgical 
data of the 217 included patients are shown in Table 1. The LGV of each type is shown in Figure 1, and the schematic diagram of each type of LGV is shown in in Figure 2. Among these, type Ia was the most prevalent (i.e., where the LGV runs between the CHA (posterior) and the CA into the portal venous system), accounting for $55.8 \%$ $(\mathrm{n}=121)$. Of the remaining patients, types II, III and IV accounted for $16.6 \%, 21.1 \%$, and $5.5 \%$, respectively $(\mathrm{n}=36,48$, and 12) (Table 2). Three patients (1.3\%) had double LGVs, and the most common types for the larger LGVs were types I ( $n=3)$. In $21(9.7 \%)$ patients, the LGV was injured during LRG, and the incidence of LGV injury hemorrhage was different among different types. Among them, type I LGV injury involved the largest number of bleeding patients, but its injury bleeding rate was only $5.8 \%$ (7/121). Although the number of type IV LGV injury bleeding patients was lower, its injury bleeding rate was as high as $41.7 \%(5 / 12)$ (Table 3$)$.

Logistic regression models were used to analyze the association between LGV bleeding and variables such as age, sex, body mass index (BMI), neoadjuvant chemoradiotherapy, type of gastrectomy, extent of LND, operation time, tumor size, tumor location, combined organ resection, $\mathrm{T}$ stage, nodes $(\mathrm{N})$ stage, total retrieved lymph node (LN), pathological stage, type of LGV, and the relationship between the LGV and peripheral arteries (Table 3). Univariate analysis revealed that the extent of LND, pathological stage, T stage, and type of LGV were significantly associated with LGV injury and hemorrhage $(\mathrm{P}<0.05)$. Multivariate analysis showed that enlarged LND [D2 vs. D2+, odds ratio (OR) $=11.841,95 \%$ CI: $3.908-$ 35.880 ], late $\mathrm{T}$ stage [postoperative tumor $(\mathrm{pT}) \leq 2 \mathrm{vs}$. $\mathrm{pT}$ $>2, \mathrm{OR}=3.076,95 \%$ CI: 1.078-8.774], late pathological stage (0/I/II vs. III/IV, OR=3.227, 95\% CI: $1.597-6.519)$, and type IV LGV (yes $v s$. no, OR $=15.856,95 \%$ CI: 4.92151.083) were independent risk factors for LGV injury hemorrhage (Table 4).

None of the 16 patients with LGV injury-induced bleeding were transferred to laparotomy due to uncontrolled hemorrhage. Furthermore, none of the patients had postoperative bleeding-related complications, and all were successfully discharged after the operation.

\section{Discussion}

As one of the important veins in the upper margin of the pancreas, the LGV is particularly important in LND during LRG. However, it has a wide range of variations and diversified courses. The root of the LGV is mainly converged in the dorsal part of the pancreas and is difficult to expose. Therefore, most surgeons rarely actively expose the root of the LGV during LND. Previous studies of the anatomical relationships around the LGV have focused on both anatomy and radiology. It was reported that $48(57.50 \%)$ of 80 cadavers exhibited a LGV that converged into the PV behind the CHA or hepatic artery (PHA). In 23 cases $(28.75 \%)$, the LGV ran to the SV between the back of the pancreatic body and the front of the SA. Three cases $(3.75 \%)$ of LGV were remitted to the SV after the SA. In eight cases (10.0\%), the LGV ran behind the pancreatic body and in front of the CHA or the SA into the PV angle (9). Kawasaki et al. (10) retrospectively analyzed the abdominal computer tomography (CT) data of 81 patients. The results showed that 40 patients (49.4\%) had LGVs that ran behind the CHA or the PHA, 18 patients (22.2\%) had LGVs that ran in front of the CHA, 17 patients $(21.0 \%)$ had LGVs that ran in front of the SA, two patients $(2.5 \%)$ had LGVs that ran in front of the SA, and four patients (4.9\%) had LGVs that ran in other parts. It has also been reported that the LGV converges directly into the liver or the superior mesenteric vein (SMV) $(11,12)$.

Zhi et al. (13) first summarized the anatomical relationship between the LGV and peripheral arteries (CHA/SA) and the pancreas of 100 patients who underwent LRG in China. They found that the LGV walking behind the CHA (45.5\%, 40 cases) was the most common anatomical type. However, Lee et al. (14) observed the videos of 405 patients who underwent LRG and found that there were six main anatomic relationships between the LGV and peripheral arteries [CHA/SA/left gastric artery (LGA)] and the pancreas. The LGV running behind the CHA accounted for $48.1 \%$ ( $n=195)$. In LRG, whether it be total, distal, or proximal gastrectomy, involves the dissection of the No. 7, 8a, 9, and 11p LNs. When dissecting the LNs around the CHA, SA, and CA, LGV is around these arteries and is easily injured (resulting in bleeding). The LGA is the most important blood vessel of the stomach. When LNs are dissected, the LGA must be cut off, and the CA, CHA, SA must be preserved. Therefore, the LGA has less influence on the injury to the LGV, and analysis of the relationship between the LGV and peripheral arteries (CA/CHA/SA) and the pancreas is of greater clinical significance in this study. At the same time, we found that the LGV running between the CHA (posterior) and the CA into the portal venous system is the most common anatomical type. 
Table 1 Clinicopathological characteristics and surgical data

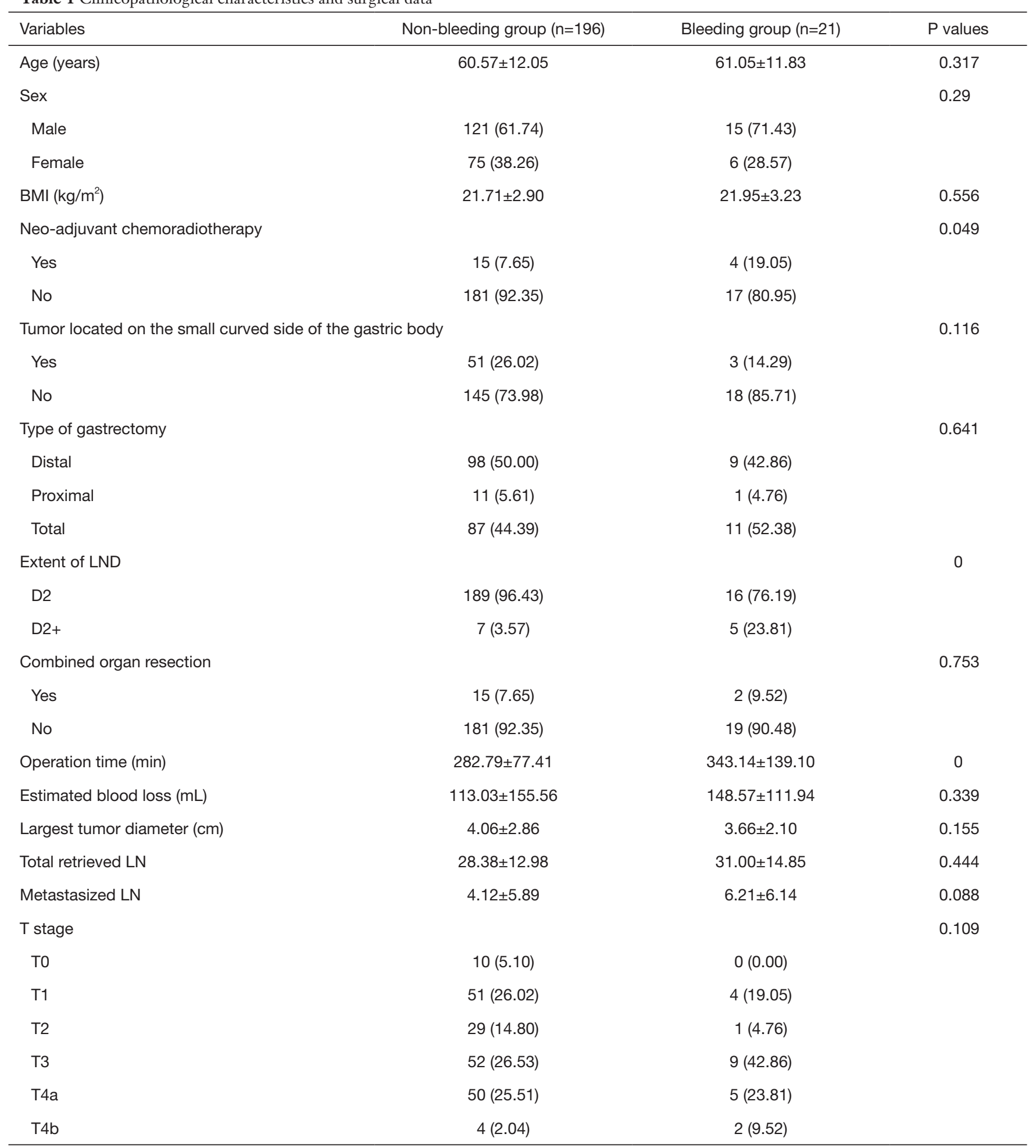

Table 1 (continued) 
Table 1 (continued)

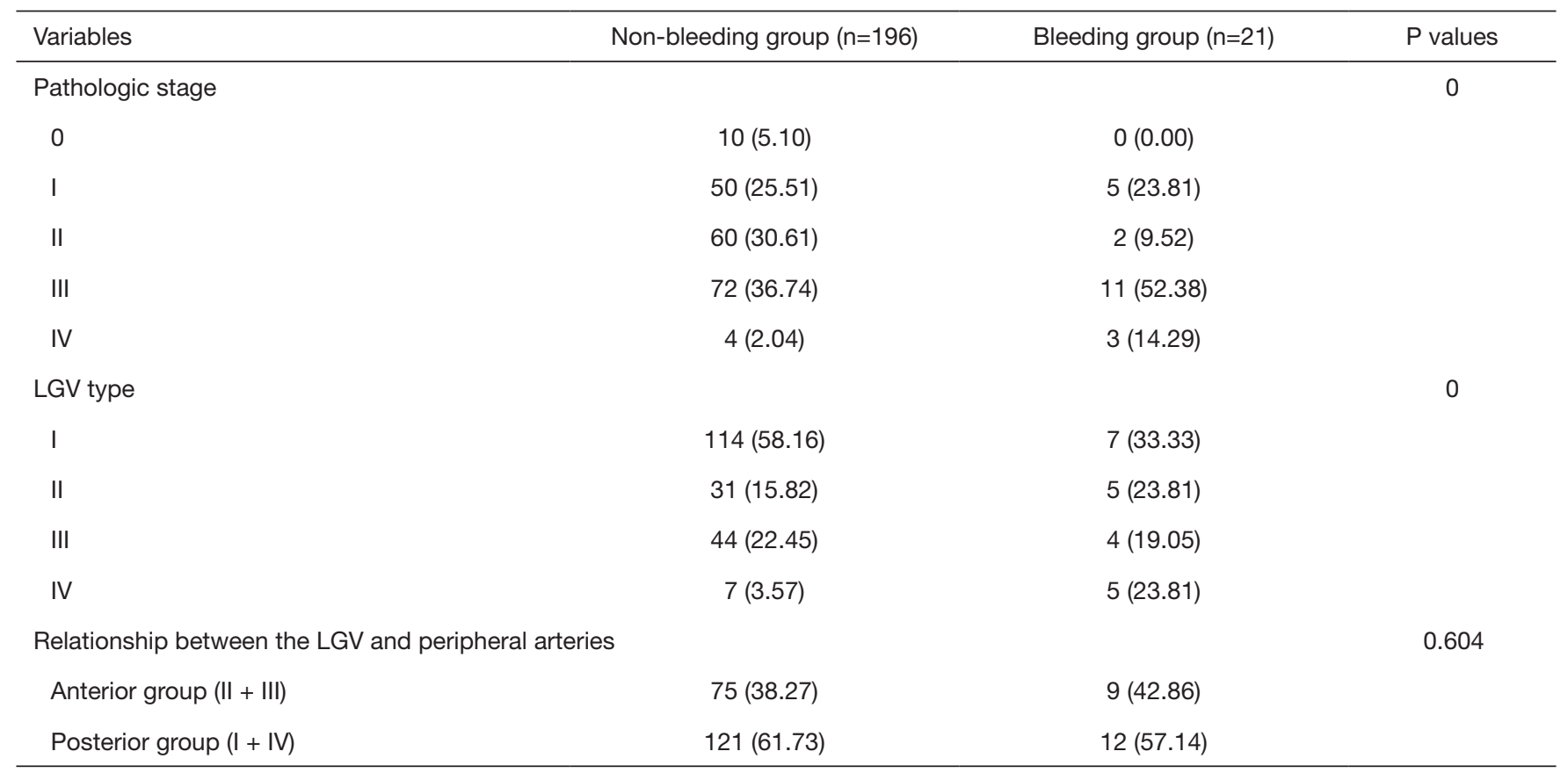

Data are shown as mean \pm SD or number (percentage). Pathologic staging according to the 2010 International Anti-Cancer Alliance (UICC) version 7 standard (8). BMI, body mass index; LNs, lymph nodes; LND, lymph node dissection; LGV, left gastric vein.
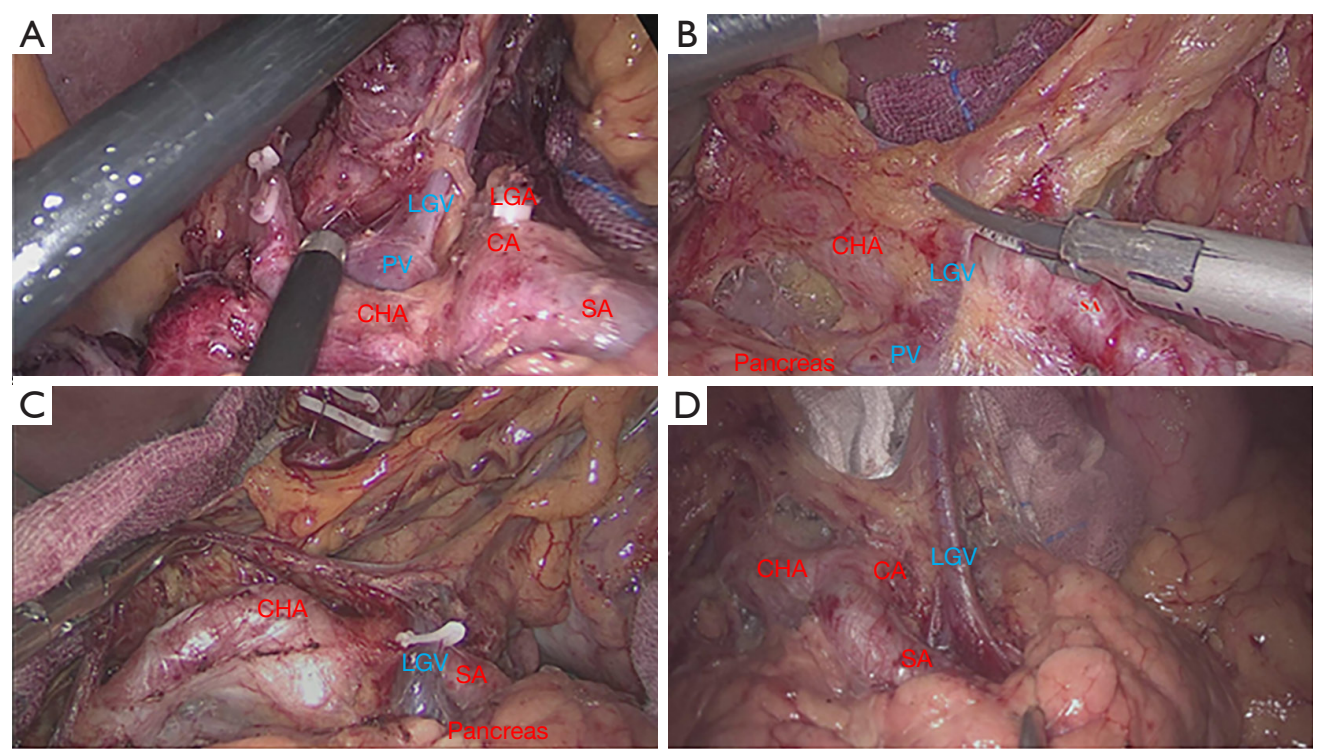

Figure 1 Various LGV types observed during LRG. Type I: the LGV runs between the CHA (posterior) and the CA into the portal venous system; Type II: the LGV runs between the pancreatic body (posterior) and the CHA (anterior) into the portal venous system; Type III: the LGV runs between the pancreatic body (posterior) and the SA (anterior) into the portal venous system; Type IV: the LGV runs between the SA (posterior) and the CA into the portal venous system. CA, celiac artery; LGA, left gastric artery; CHA, common hepatic artery; SA, splenic artery; SV, splenic vein; LGV, left gastric vein. 

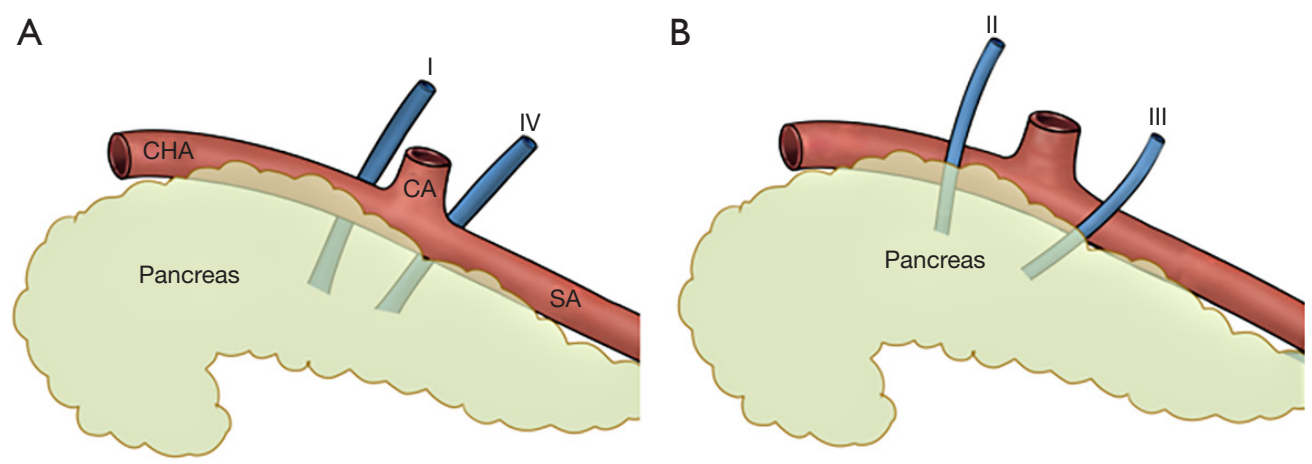

Figure 2 Course of the LGV identified by the anatomic relations to the peripheral arteries and the pancreas. I, II, III, IV, If the patient has two or more LGVs, the type of LGV is determined by the largest branch.

Table 2 Anatomical type and frequency of the LGV

\begin{tabular}{|c|c|c|}
\hline Variation type & Description & Frequency \\
\hline II & LGV runs between the pancreatic body (posterior) and the CHA (anterior) into the portal venous system & $16.6 \%(n=36)$ \\
\hline III & LGV runs between the pancreatic body (posterior) and the SA (anterior) into the portal venous system & $21.1 \%(n=18)$ \\
\hline IV & LGV runs between the SA (posterior) and the CA into the portal venous system. & $5.5 \%(n=12)$ \\
\hline
\end{tabular}

CA, celiac artery; CHA, common hepatic artery; SA, splenic artery; LGV, left gastric vein.

Table 3 Comparison of anatomical types of LGV with bleeding

\begin{tabular}{lc}
\hline Variables & $\begin{array}{c}\text { Number and frequency of } \\
\text { LGV in injury bleeding (n/total) }\end{array}$ \\
\hline I & $5.8 \%(7 / 121)$ \\
II & $13.9 \%(5 / 36)$ \\
III & $8.3 \%(4 / 48)$ \\
IV & $41.7 \%(5 / 12)$ \\
\hline
\end{tabular}

LGV, left gastric vein.

In this study, the incidence of hemorrhage in LGV injury was $9.7 \%(n=21)$, while that of IV LGV being easily injured (resulting in bleeding) was as high as $41.7 \%(5 / 12)$. Lee et al. (14) found that 49 cases (13.0\%) had bleeding from LGV injury, while the LGV had a higher risk of bleeding from injury before or after the SA $(17.8 \%)$, which might be due to the increased anatomical difficulty caused by the hunched uplift of the pancreas. The LGV, which runs in the anterior part of the LGA, has the lowest probability of hemorrhage, which may be related to the easy detection of the LGV and the simple dissection of LNs. However, according to the risk factor analysis of LGV injury, LGV injury hemorrhage was not associated with the relationship between the LGV and peripheral arteries. If a LGV runs between the CHA/ $\mathrm{SA}$ (posterior) and the CA into the portal venous system, it will increase the difficulty of the LRG operation. It is possible that the LGV entry point on the posterior aspect of the artery and the hunched bump of the pancreas were obscured, thus increasing the difficulty of the procedure. Therefore, for a LGV that runs behind the CHA into the portal venous system, we choose to actively expose the root of the PV and the LGV below the angle between the CHA and the gastroduodenal artery (GDA) during the dissection of LNs, which can effectively reduce the injury and hemorrhage of LGV. In this study, type I LGV injury has the largest number of bleeding patients, but its injury bleeding rate was only $5.8 \%(7 / 121)$.

The extent of LND and pathological stage were found to be significantly associated with LGV injury and hemorrhage $(\mathrm{P}<0.05)$. Patients undergoing extended 
Table 4 Univariate and multivariate analyses of the risk factors for LGV injury bleeding

\begin{tabular}{|c|c|c|c|c|}
\hline Variables & \multicolumn{2}{|c|}{ Univariate } & \multicolumn{2}{|c|}{ Multivariate } \\
\hline Age (<60 vs. $\geq 60$ year) & $1.137(0.408-3.171)$ & 0.806 & & \\
\hline Sex (male vs. female) & $1.115(0.276-4.507)$ & 0.879 & & \\
\hline $\mathrm{BMI}\left(<24\right.$ vs. $\left.\geq 24 \mathrm{~kg} / \mathrm{m}^{2}\right)$ & $0.833(0.291-2.384)$ & 0.733 & & \\
\hline Total gastrectomy (yes vs. no) & $1.445(0.507-4.114)$ & 0.491 & & \\
\hline $\begin{array}{l}\text { Tumor located on the small curved side of } \\
\text { the gastric body (yes vs. no) }\end{array}$ & $0.449(0.618-9.706)$ & 0.202 & & \\
\hline Extent of LND (D2 vs. D2+) & $0.069(0.012-0.401)$ & 0.003 & $11.841(3.908-35.880)$ & 0.000 \\
\hline Total retrieved LN ( $\geq 40$ vs. $<40$ ) & $1.057(0.038-3.603)$ & 0.930 & & \\
\hline Pathological stage (0, I, II vs. III, IV) & $0.310(0.153-0.626)$ & 0.001 & $3.227(1.597-6.519)$ & 0.001 \\
\hline Operation time ( $<240$ vs. $\geq 240 \mathrm{~min})$ & $1.131(0.384-3.332)$ & 0.823 & & \\
\hline Largest tumor diameter $(<5$ vs. $\geq 5 \mathrm{~cm})$ & $1.085(0.520-2.264)$ & 0.829 & & \\
\hline Type IV LGV (yes vs. no) & $0.119(0.042-0.334)$ & 0.000 & $15.856(4.921-51.083)$ & 0.000 \\
\hline $\begin{array}{l}\text { Relationship between the LGV and peripheral arteries } \\
\text { (anterior group (II + III) vs. posterior group }(I+I V)\end{array}$ & $0.826(0.342-1.624)$ & 0.580 & & \\
\hline
\end{tabular}

LGV, left gastric vein; BMI, body mass index; LN, lymph node; LND, lymph node dissection.

LND (D2+) tended to have a late pathological stage, and there may have also been more LN metastasis around the $\mathrm{CA}$ and its branches. Therefore, the surgical difficulty increases accordingly, which increases the risk of bleeding from perigastric vascular injury, including the LGV. Among the 21 patients with LGV injury and hemorrhage, five underwent enlarged LND (D2+), including two patients who received omental sac excision and three patients who received para-aortic LND. The operative time and dissection of positive LNs were also higher in the non-bleeding group. Similarly, later pathological stage was considered as an independent risk factor for LGV lesion bleeding during LRG (OR $=1.87,95 \%$ CI: $0.81-0.4 .38)$ (14). T staging of gastric cancer directly affects the surgical difficulty, and $T$ stage was found to be an independent risk factor for bleeding from LGV injury. A T stage of T3 or T4 will limit the pull and exposure of the operation, thus increasing the difficulty of LND. Also, a tumor located on the small curved side of the gastric body will further increased the difficulty of No.7, 8a, and 9 LNDs, thereby increasing the risk of LGV injury and bleeding. However, in this study, the injury and bleeding of LGV was found to be independent of tumor location. In addition, the metastasis of No. 7, 8a, and 9 LNs in $\mathrm{N}$ staging also affected the dissection of the LGV. However, this was a retrospective study, and No. 7, 8a, and 9 LNs were divided into independent groups in the previous surgery.

Certainly, LGV injury and hemorrhage were also related to the degree to which the main surgeon was familiar with the anatomy of the perigastric vessels under laparoscopy, as well as the traction, exposure of assistant, laparoscopic system, and surgical instruments, etc. At present, the impact of bleeding from LGV injury on the outcome of the procedure is unknown. Although the operation time of patients with LGV injury bleeding under laparoscopy is prolonged and intraoperative blood loss is increased, in addition to anatomical location of LGV, numerous factors 
determine the surgical results. Therefore, further clinical study is required to identify methods of reducing the effect of LGV injury on the operation.

Studies have shown that preoperative selection of three-dimensional reconstruction can detect anatomical abnormalities in perigastric vessels and reduce the difficulty of LND (15-18). Miyamoto et al. (15) reported that for 84 patients who underwent LRG, preoperative threedimensional reconstruction of the perigastric vessels was helpful in reducing intraoperative hemorrhage. It can be seen that preoperative three-dimensional reconstruction can effectively avoid vascular injury and reduce intraoperative hemorrhage. However, 3D CT angiography (CTA) is expensive and it requires a large amount of contrast agent Further study is needed to demonstrate that preoperative three-dimensional reconstruction technology can improve the safety of LRG, and thus, it has not been widely used in clinical practice.

This study had some limitations that should be noted. Firstly, this was a retrospective, single-center study with a small number of cases included. Secondly, we chose to actively expose the root of the PV and the LGV below the angle between the CHA and the GDA while also cleaning up the NO.8p LNs, whether it would increase the number of positive lymph nodes. The survival prognosis of patients also requires further research.

\section{Conclusions}

LGVs that run between the CHA (posterior) and the $\mathrm{CA}$ into the portal venous system are the most common anatomical type. A LGV that runs between the SA (posterior) and the CA into the portal venous system is easily injured (resulting in bleeding). LGV injury and hemorrhage are affected by a variety of factors, and therefore, careful intraoperative dissection is necessary to avoid damage to the LGV.

\section{Acknowledgments}

Funding: The Guangdong Provincial Hospital of Traditional Chinese Medicine Science and Technology Research Project (YN2016ZD02); the Clinical Research Project of Guangdong Provincial Hospital of Traditional Chinese Medicine (1010) (YN1010911); General Items of Scientific Research Projects of Guangdong Administration of Traditional Chinese Medicine (No. 20191159).

\section{Footnote}

Reporting Checklist: The authors have completed the STROBE reporting checklist. Available at https://dx.doi. org/10.21037/jgo-21-352

Data Sharing Statement: Available at https://dx.doi. org/10.21037/jgo-21-352

Conflicts of Interest: All authors have completed the ICMJE uniform disclosure form (available at https://dx.doi. org/10.21037/jgo-21-352). The authors have no conflicts of interest to declare.

Etbical Statement: The authors are accountable for all aspects of the work in ensuring that questions related to the accuracy or integrity of any part of the work are appropriately investigated and resolved. All procedures performed in this study involving human participants were in accordance with the Declaration of Helsinki (as revised in 2013). The study was approved by regional ethics board of Guangdong Provincial Hospital of Chinese Medicine (NO: ZE2021-173-01). Individual consent for this retrospective analysis was waived.

Open Access Statement: This is an Open Access article distributed in accordance with the Creative Commons Attribution-NonCommercial-NoDerivs 4.0 International License (CC BY-NC-ND 4.0), which permits the noncommercial replication and distribution of the article with the strict proviso that no changes or edits are made and the original work is properly cited (including links to both the formal publication through the relevant DOI and the license). See: https://creativecommons.org/licenses/by-nc-nd/4.0/.

\section{References}

1. Kim HH, Hyung WJ, Cho GS, et al. Morbidity and Mortality of Laparoscopic Gastrectomy Versus Open Gastrectomy for Gastric Cancer: An Interim ReportA Phase III Multicenter, Prospective, Randomized Trial (KLASS Trial). Ann Surg 2010;251:417-20.

2. Kim HH, Han SU, Kim MC, et al. Long-Term Results of Laparoscopic Gastrectomy for Gastric Cancer: A Large-Scale Case-Control and Case-Matched Korean Multicenter Study. J Clin Oncol 2014;32:627-33.

3. Kim YW, Baik YH, Yun YH, et al. Improved quality of life 
outcomes after laparoscopy-assisted distal gastrectomy for early gastric cancer: results of a prospective randomized clinical trial. Ann Surg 2008;248:721.

4. Yuasa Y, Okitsu H, Goto M, et al. Three-dimensional CT for preoperative detection of the left gastric artery and left gastric vein in laparoscopy-assisted distal gastrectomy. Asian J Endosc Surg 2016;9:179-85.

5. Wottawa CR, Cohen JR, Fan RE, et al. The role of tactile feedback in grip force during laparoscopic training tasks. Surg Endosc 2013;27:1111-8.

6. Natsume T, Shuto K, Yanagawa N, et al. The classification of anatomic variations in the perigastric vessels by dual-phase CT to reduce intraoperative bleeding during laparoscopic gastrectomy. Surg Endosc 2011;25:1420-4.

7. Association JGC. Japanese gastric cancer treatment guidelines 2010 (ver. 3). Gastric Cancer 2011;14:113-23.

8. Cuccurullo V, Mansi L. AJCC Cancer Staging Handbook: from the AJCC Cancer Staging Manual (7th edition). Eur J Nucl Med Mol Imaging 2011;38:408.

9. Liu YF, Xu ED. Local anatomy of the left gastric vein and clinical significance. Journal of Clinical Applied Anatomy $1983 ;(1): 47$.

10. Kawasaki K, Kanji S, Kobayashi I, et al. Multidetector computed tomography for preoperative identification of left gastric vein location in patients with gastric cancer. Gastric Cancer 2010;13:25-9.

11. Kuwada K, Kuroda S, Kikuchi S, et al. Strategic approach to concurrent aberrant left gastric vein and aberrant

Cite this article as: Zhu $\mathrm{X}$, Zhao Q, Xiong W, Luo L, Zheng Y, Huang H, Li J, Wan J, Xie W, Wang W. Anatomical observation and clinical significance of the left gastric vein in laparoscopic radical gastrectomy. J Gastrointest Oncol 2021;12(4):1407-1415. doi: 10.21037/jgo-21-352 left hepatic artery in laparoscopic distal gastrectomy for early gastric cancer: A case report. Asian J Endosc Surg 2015;8:454-6.

12. Feng XY, Chen YB, Chen S, et al. Relationship between the number of lymph node detection and prognosis in stage II gastric cancer after D(2) dissection. Zhonghua Wei Chang Wai Ke Za Zhi 2010;13:346-9.

13. Zhi PK, Zhang C, Yu J, et al. Laparoscopic anatomy and clinical significance of perigastric vessels. Chinese Journal of Clinical Anatomy 2012;30:149-52.

14. Lee H, Lee J. Anatomic variations in the left gastric vein and their clinical significance during laparoscopic gastrectomy. Surg Endosc 2018;33:1903-9.

15. Miyamoto R, Inagawa S, Nagai K, et al. Threedimensional reconstruction of vascular arrangement including the hepatic artery and left gastric vein during gastric surgery. SpringerPlus 2016;5:835.

16. Wu Y, Chen G, Wu P, et al. CT imaging-based determination and classification of anatomic variations of the left gastric vein. Surg Radiol Anat 2017;39:249-55.

17. Iino I, Sakaguchi T, Kikuchi H, et al. Usefulness of three-dimensional angiographic analysis of perigastric vessels before laparoscopic gastrectomy. Gastric Cancer 2013;16:355-61.

18. Miyaki A, Imamura K, Kobayashi R, et al. Preoperative assessment of perigastric vascular anatomy by multidetector computed tomography angiogram for laparoscopy-assisted gastrectomy. Langenbecks Arch Surg 2012;397:945-50. 